\title{
Towards Quantum Entanglement in Nanoelectromechanical Devices
}

\author{
J. Eisert, ${ }^{1,2}$ M. B. Plenio, ${ }^{2}$ S. Bose, ${ }^{3,4}$ and J. Hartley ${ }^{2}$ \\ ${ }^{1}$ Institut für Physik, Universität Potsdam, Am Neuen Palais 10, D-14469 Potsdam, Germany \\ ${ }^{2}$ QOLS, Blackett Laboratory, Imperial College London, Prince Consort Road, London SW7 2BW, United Kingdom \\ ${ }^{3}$ Department of Physics and Astronomy, University College London, Gower Street, London, WC1E 6BT, United Kingdom \\ ${ }^{4}$ Institute for Quantum Information, California Institute of Technology, Pasadena, California 91125, USA
}

(Received 26 December 2003; published 4 November 2004)

\begin{abstract}
We study arrays of mechanical oscillators in the quantum domain and demonstrate how the motions of distant oscillators can be entangled without the need for control of individual oscillators and without a direct interaction between them. These oscillators are thought of as being members of an array of nanoelectromechanical resonators with a voltage being applicable between neighboring resonators. Sudden nonadiabatic switching of the interaction results in a squeezing of the states of the mechanical oscillators, leading to an entanglement transport in chains of mechanical oscillators. We discuss spatial dimensions, $Q$ factors, temperatures and decoherence sources in some detail, and find a distinct robustness of the entanglement in the canonical coordinates in such a scheme. We also briefly discuss the challenging aspect of detection of the generated entanglement.
\end{abstract}

DOI: 10.1103/PhysRevLett.93.190402

PACS numbers: 03.65.Ud, 03.65.Yz, 03.67.Mn, 07.10.Cm

In 1959, Feynman suggested in a famous talk that it appears to be a fruitful enterprise to think about manipulating and controlling mechanical devices at a very small scale. Since then, the study of microelectromechanical and even nanoelectromechanical systems (NEMS) has developed into a mature field of research [1-4]. Mechanical oscillators with spatial dimensions of a few nanometers and very high frequencies can now be routinely manufactured. Applications of such NEMS range from mechanically detected magnetic resonance imaging, to sensing of biochemical systems, and to ultrasensitive probing of thermal transport. Indeed, the NEMS devices that are presently manufactured in experimental studies are close to or already on the verge of the quantum limit [1-4]. While first quantum effects are already being observed and studied, it is interesting to see to what extent it is feasible to prepare nanoscale mechanical oscillators in states where the quantum nature becomes most manifest: in states that are genuinely entangled in the canonical coordinates of position and momentum. This can be interesting, as it provides another stepping stone towards quantum state control and quantum information processing in mechanical systems. They would therefore also permit the exploration of the limiting region between the quantum and the classical world. This might be facilitated by another application of entanglement, namely, its use to enhance quantum measurement schemes where entangled states represent a very sensitive probe.

The key question that will be addressed in this Letter is how it is possible to entangle mechanical oscillators well separated in space, without the need for making them interact directly and with a minimum need for individual local control which is difficult to achieve at the nanolevel. This will be accomplished by triggering squeezing and entanglement by a global nonadiabatic change of the interaction strength in a linear array of oscillators, but without individually addressing any of the oscillators of the array. In this way, one can achieve long-range entanglement that will persist over length scales that are much larger than the typical entanglement length for the ground state of the system [5]. The physics underlying this approach, especially the nonadiabaticity requirement, will be discussed in more detail later on. Several schemes to probe quantum coherence of mechanical resonators in different setups and situations have been proposed so far $[6,7]$. Notably, while the earlier proposal of entangling macroscopic oscillators [7] entangles two adjacent oscillators in the context of a different physical setup, our scheme allows, without the need for individual local control, for entanglement in the canonical coordinates between nonadjacent (and possibly distant) microscopic oscillators by entanglement transport in a chain.

The setup that we will consider is an array of doubleclamped coupled nanomechanical oscillators as has been experimentally studied in the micromechanical realm in Ref. [8]. We assume that the beams are arranged in such a manner that between adjacent oscillators a controlled and tunable interaction can be introduced. In Ref. [8] this is experimentally achieved by applying a voltage between adjacent beams made from gold fabricated on a semiconductor membrane that are ordered alternatingly. This induces to a good approximation a nearest-neighbor interaction that can be controlled in strength. The oscillators are assumed to be cooled to temperatures such that $k T / \hbar \omega \ll 1$ with $\omega$ being the fundamental frequency of the oscillators, such that the array is operated deeply in the quantum regime. Before we discuss the time and energy scales that would be required to achieve this regime, we will exemplify the mechanism, without tak- 
ing sources of error and decoherence mechanisms into account, as we will discuss these in some detail later. We start with the Hamiltonian of $N$ quantum oscillators of mass $m$ and eigenfrequency $\omega$ ordered on a onedimensional lattice, with nearest-neighbor interaction of strength $c$. Setting $\hbar=1$ and using the $q_{k}=q_{k}^{\prime} \sqrt{m \omega}$, and $p_{k}=p_{k}^{\prime} / \sqrt{m \omega}$, where $q_{k}^{\prime}$ and $p_{k}^{\prime}$ are the canonical position and momentum of the oscillators we find $H=$ $\omega / 2 \sum_{k=1}^{N}\left[p_{k}^{2}+q_{k}^{2}(1+2 c)-2 c q_{k} q_{k+1}\right]$. For the moment, we assume for simplicity periodic boundary conditions, i.e., $q_{N+1}=q_{1}$, but this requirement will be relaxed later, and set $\omega=1$, as in this ideal treatment this merely corresponds to a rescaling of the time scale. The normal coordinates are related to the previous ones by a discrete Fourier transform, $q_{k}=\sum_{l=1}^{N} e^{2 \pi i k l / N} Q_{l} /$ $\sqrt{N}, \quad p_{k}=\sum_{l=1}^{N} e^{-2 \pi i k l / N} P_{l} / \sqrt{N}$. In these normal coordinates, satisfying $Q_{k}=Q_{N-k}^{\dagger}$ and $P_{k}=P_{N-k}^{\dagger}$, the Hamiltonian can be written in the form $H=$ $1 / 2 \sum_{k=1}^{N}\left\{P_{k} P_{k}^{\dagger}+\left[1+4 c \sin ^{2}(\pi k / N)\right]^{1 / 2} Q_{k} Q_{k}^{\dagger}\right\}$, annihilation and creation operators, and expressing the time dependent operators $Q_{k}(t)$ and $P_{k}(t)$ in terms of these operators, one arrives at the Heisenberg equations of motion for the original canonical coordinates $q_{k}(t)=$ $\sum_{r=1}^{N}\left[q_{r}(0) f_{r-k}(t)+p_{r}(0) g_{r-k}(t)\right]$ and $p_{k}(t)=\partial_{t} q_{k}(t)$, where we have defined the two functions $g_{k}(t)=$ $\sum_{l=1}^{N} e^{2 \pi i k l / N} \sin \left(\omega_{l} t\right) /\left(N \omega_{l}\right)$ and $f_{k}(t)=\partial_{t} g_{k}(t)$. In this Letter, we are dealing with states that are Gaussian, i.e., states whose characteristic function or Wigner function is a Gaussian. As such, it is completely characterized by the first and second moments [9]. The first moments will not be directly relevant for our purposes. The second moments can be arranged in the symmetric $2 N \times 2 N$-covariance matrix $\Gamma_{R, S}=2 \operatorname{Re}\langle(R-\langle R\rangle) \times$ $(S-\langle S\rangle)\rangle$, where $R$ and $S$ stand for the canonical operators $q_{1}, \ldots, q_{n}$ and $p_{1}, \ldots, p_{n}$. At this point, we assume that for times $t<0$, the oscillators are not interacting and are in the ground state. This implies that $\Gamma_{q_{n}, q_{m}}=\Gamma_{p_{n}, p_{m}}=$ $\delta_{n, m}$, and $\Gamma_{q_{n}, p_{m}}=0$, for $n, m=1, \ldots, N$.

In the setting of this Letter, we will assume that for $t<$ 0 the interaction is switched off and the system is in its ground state and time-independent. At time $t=0$ the interaction is then switched on instantaneously to ensure nonadiabaticity and consequently the system is out of equilibrium and evolving in time for $t>0$ according to the equations of motion for the second moments given by $\Gamma_{q_{n}, q_{m}}(t)=\left[a_{n, m}(t)+d_{n, m}(t)\right] / 2, \quad \Gamma_{q_{n}, p_{m}}(t)=$ $\left[b_{n, m}(t)+e_{n, m}(t)\right] / 2, \quad \Gamma_{p_{n}, p_{m}}(t)=\left[c_{n, m}(t)+a_{n, m}(t)\right] / 2$, where $a_{n, m}=\sum_{k=1}^{N} f_{k-n} f_{k-m}, b_{n, m}=\partial_{t} a_{m, n} / 2, c_{n, m}=$ $\sum_{k=1}^{N} \partial_{t} f_{k-n} \partial_{t} f_{k-m}, \quad d_{n, m}=\sum_{k=1}^{N} g_{k-n} g_{k-m}, \quad$ and $e_{n, m}=\partial_{t} d_{n, m} / 2$.

Before we discuss in detail the nonadiabaticity requirement and other idealizations as well as the physics behind this approach we demonstrate the success of the approach. We are now in the position to study the entanglement of two very distant oscillators when we trace out all the others. The chain is translationally invariant, and hence, a single oscillator, say labeled 1 , can be singled out, and we may look at the degree of entanglement as a function of time and discrete distance. We quantify entanglement in terms of the logarithmic negativity, defined as $E_{N}(\rho)=\log \left\|\rho^{T_{A}}\right\|_{1}$ for states $\rho$, where $\rho^{T_{A}}$ is the partial transpose and $\|\cdot\|_{1}$ denotes the trace-norm. This quantity is an upper bound for distillable entanglement and has an interpretation of an asymptotic preparation cost [10].

Before we consider the entanglement created in this way, let us first remind ourselves about the entanglement structure of the ground state of the harmonic lattice Hamiltonian: there, the bi-partite entanglement between two distinguished oscillators is only nonzero for nearest neighbors. Next-to-nearest neighbors are already separable for all parameters, as are more distant oscillators, even in case of an arbitrarily large correlation length of the chain when approaching criticality [5].

This is very much in contrast to the situation encountered here: Astonishingly indeed, we find that even very distant oscillators become significantly entangled over time. This dependence is depicted in Fig. 1. For a time interval $\left[0, t_{0}\right), t_{0}>0$, the state of the oscillators with labels 1 and $n$ is separable, then, for $t>t_{0}$ it becomes entangled. This time $t_{0}$ is approximately given by $t_{0} \approx$ $n /(2 \gamma \Omega)$, There is what can be called a finite "speed of propagation" of the quantum correlations, which is in fact closely related to the speed of sound in this chain.
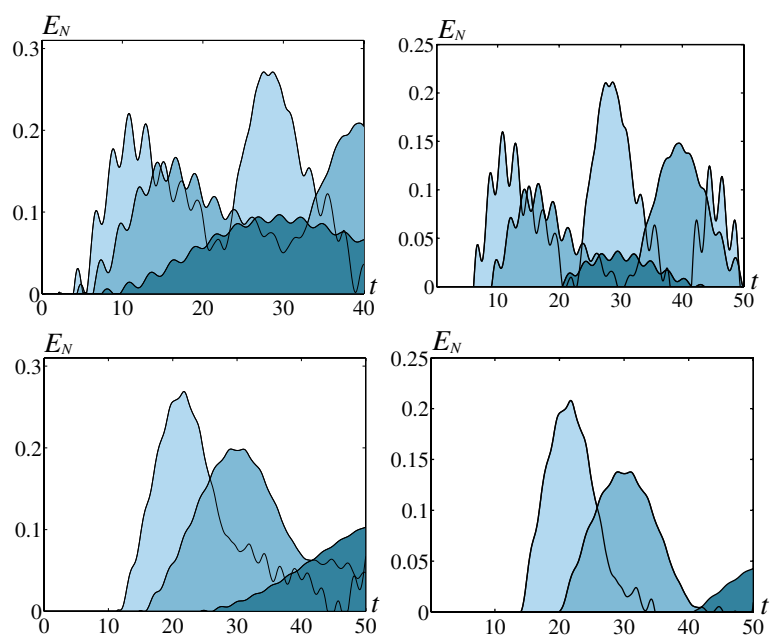

FIG. 1 (color online). The degree of entanglement as a function of time in a chain of length 8 with open boundary conditions. Depicted in light, medium, and dark grey are the curves for the values $c=0.3, c=0.2$, and $c=0.1$ in the above units. The figures in the lower row depict the entanglement between the first and the last oscillator in an open chain, for the upper row the two diametrically opposed oscillators in a ring are considered. The figures on the left corresponds to the noiseless case. The figures on the right show the degree of entanglement under the decoherence and for nonzero temperature. Values are chosen that correspond to the $Q$ factor $Q=10^{3}$, fundamental frequency $5 \mathrm{GHz}$, and temperature of $10 \mathrm{mK}$. 
The amount of entanglement roughly falls off as $1 / n$, but becomes strictly zero after a finite distance. For $c=0.1$, for example, this happens for $n$ larger than 500. This long-range nature of the entanglement is remarkable.

The central idea behind the method above is the fact that an instantaneous change in the potential of a single harmonic oscillator in its ground state will generally make its state time dependent and squeezed. In the same way a change in the coupling strength between oscillators drives the systems away from equilibrium. In the course of the subsequent time evolution the squeezing is then transformed into entanglement due to the nearestneighbor coupling. The origin for this is the fact that time evolution is described by a Hamiltonian quadratic in the canonical coordinates and therefore has an effect analogous to passive optical elements. Finally, this entanglement propagates, as every other excitation, through the chain and can therefore lead to entanglement.

In any realistic setting, this switching can not be instantaneous, and an important question is how fast the switching process must be in order to generate significant entanglement. Figure 2 depicts the amount of entanglement in the first maximum when the interaction strength is linearly increased over a time interval $\left[0, t^{\prime}\right]$. We find that for times $t^{\prime}<1$, any nonzero switching time is unproblematic. This is because the change in coupling strength is faster than any eigenfrequency in the system, preventing an adiabatic following. For very slow switching, $t^{\prime} \gg 1$, most entanglement is lost because the system can adiabatically follow the parameter change.

Let us now turn to the discussion of a realisation in NEMS of such an array. Presently, NEMS made from $\mathrm{SiC}$ have been manufactured experimentally with frequencies around $1-10 \mathrm{GHz}$, with spatial dimensions of the order of $10 \mathrm{~nm}$ [1-4]. Doubly clamped beams have the advantage of higher fundamental frequencies with the same spatial dimensions. The $Q$ factors for NEMS of these dimensions achieve values of significantly more than $Q=$ $10^{3}$ [11]. Concerning the extent to which the ground state can initially be reached, cooling of the oscillators to $10 \mathrm{mK}$ seems feasible [12] using a helium dilution refrigerator [1], [4] (for the possibility of the equivalent of laser cooling to the ground state, see Ref. [13]).

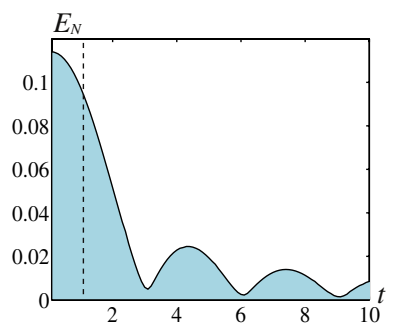

FIG. 2 (color online). Maximum degree of entanglement between the end points as a function of the switching time $t^{\prime}$ in the above units for a chain of length eight and $c=0.1$. The dotted line represents the unit frequency of a free oscillator.
Decoherence mechanisms cannot be entirely avoided in a quantum system so close to macroscopic dimensions. After all, $Q$ factors describe nothing but the coupling strength to external degrees of freedom beyond our control. Most of the dissipation and decoherence is expected to be due to the coupling with the degrees of freedom of the substrate to which the resonator is connected. Let us now specify the decoherence model [14]: In the setting described here, we are not in the high temperature limit, but close to zero temperature. Secondly, we do not have product initial conditions: in a realistic setting, the chain and the environment are initially not in a completely uncorrelated state, but rather in the Gibbs state of the coupled joint system, and then driven away from equilibrium [15]. We have hence modeled the decoherence process by appending local heat baths consisting of a finite number $M$ of modes to each of the oscillators with canonical coordinates $q_{j}^{k}, p_{j}^{k}$ for $k=1, \ldots, M$. We choose an Ohmic spectral density in which case the Langevin equation for the Heisenberg picture position becomes the one of classical Brownian motion in the classical limit; i.e., the coupling is specified by the Hamiltonian $H_{j}=$ $\zeta \tilde{\omega}_{j}\left(q_{j} \otimes \sum_{i=1}^{M} q_{j}^{i}\right)$, where $\tilde{\omega}_{j}=j \Lambda / M$, and $\Lambda>0$ is a cutoff frequency. This Hamiltonian induces decoherence and dissipation, and the number $\zeta>0$ has in our analysis been chosen in such a manner that the energy dissipation rate reflects exactly the rate $1 / Q$ corresponding to the experimentally found $Q$ factors (see, e.g., Refs. [1,17]). With this value of $\zeta$, the initial state before switching on the interaction is the Gibbs state of the canonical ensemble of the whole chain including the appended heat baths. The resulting map is nevertheless a Gaussian operation, such that it is sufficient to know the second moments to specify entanglement properties. This model grasps in the simplest possible manner the various noise processes [18] in NEMS.

The plot on the right-hand side of Figure 1 depicts the behavior of the degree of entanglement for system parameters that are close to those used in actual experimental settings. We see that the scheme is surprisingly robust against noise processes and nonzero temperatures. Comparably low $Q$ factors are not particularly harmful given the large speed of propagation; yet too high temperatures, turn the correlations into merely classical ones. This effect is evidently more harmful for longer chains. Notably, for two oscillators, quite large values of the degree of entanglement can be achieved. For example, for a two-oscillator system, with system parameters as in the plot at the right of Fig. 1, the degree of entanglement as quantified in terms of the log-negativity reaches values larger than 0.6 for $c=0.4$. Assuming the ability to cool to $10 \mathrm{mK}$, oscillators with fundamental frequencies of $2 \mathrm{GHz}$ would be sufficient to generate entanglement. This would be the most feasible starting point.

The most significant technological challenge in an experimental realization of this scheme (and actually 
any scheme that involves entanglement in the canonical coordinates of oscillators at the nanoscale) is the actual detection of entanglement. We would need to couple the two chosen oscillators to canonical coordinate transducers whose output is proportional to position and momentum, which is fed into an amplifier that produces a classical signal [19]. What has to be measured with very high sensitivity are the second moments of the canonical coordinates $q_{m}, q_{n}, p_{m}$, and $p_{n}$, i.e., covariance matrix elements. If not all entries can be assessed, bounds of the type $E_{N}(\rho) \geq \max \left\{0,-\log \left[\left(\left\langle\left(q_{n}-q_{m}\right)^{2}\right\rangle+\left\langle\left(p_{n}+p_{m}\right)^{2}\right\rangle\right) /\right.\right.$ $2)]\}$ may be used to estimate the degree of entanglement. If only a position transducer is available, stroboscopic measurements may be employed where only two measurements per cycle are performed (note that $p_{m}(t=0)=$ $q_{m}(t=\pi / 4)$ and position and momentum are interchanging roles with frequency $\omega$ ) [19]. Alternatively, continuous single-transducer measurements may be performed which make use of only a position transducer and a sinusoidally modulated output [19]. This leaves us with the problem of measuring position and momentum with great accuracy: conventional optical transducers are not applicable in NEMS, but near-field optical sensors or piezoelectric detectors may be used [1]. References [3,20] describe and make use of a balanced electronic detection scheme of displacement. The most promising to date appears to be a capacitive coupling of an electrode placed on a resonator to the gate of a single-electron transistor, as studied theoretically in Ref. [21] and experimentally in Refs. [2,22]. The sensitivity reached in such setups is rapidly increasing, and is presently about a factor of 4.3 away from the quantum limit of the considered oscillator [22], while this factor was still about a 100 a year ago, and it is argued that with these techniques, the quantum limit could well be reached in the not too far future $[2,12,21,22]$. Note that the chain of mechanical oscillators may also be used as a quantum channel [23].

We have presented a method of entangling mechanical oscillators on the nanoscale which are located at macroscopically different locations at the ends of a chain, without the need of addressing each of the oscillators in the chain. We have introduced the suggested setup formally, and have discussed issues of decoherence and measurement. As such, the scheme is not yet a fully feasible scheme ready for experimental implementation. Yet, it is the hope that this letter can point towards significant next steps that could be taken when further exploring the quantum domain with nanoelectromechanical devices.

This research was partly triggered by an inspiring talk given by M. Roukes at CalTech. J. E. would like to thank J. Preskill and his IQI group at CalTech for kind hospitality during a research visit and S. B. would like to thank IQI for financial support. M. B. P. is supported by the Royal Society. We would like to thank K. Schwab, M. Roukes, I. Wilson-Rae, P. Rabl, and C. Henkel for interesting communication. This work has been supported by the EU
(QUPRODIS), the DFG, the U.S. Army (DAAD 19-020161), and the EPSRC QIP-IRC.

[1] M. Roukes, Phys. World 14, 25 (2001); H. G. Craighhead, Science 290, 1532 (2000).

[2] R. G. Knobel and A. N. Cleland, Nature (London) 424, 291 (2003).

[3] X. M. H. Huang, C. A. Zorman, M. Mehregany, and M. L. Roukes, Nature (London) 421, 496 (2003).

[4] K. C. Schwab, E. A. Henriksen, J. M. Worlock, and M. L. Roukes, Nature (London) 404, 974 (2000).

[5] K. Audenaert, J. Eisert, M. B. Plenio, and R. F. Werner, Phys. Rev. A 66, 042327 (2002).

[6] A. D. Armour, M. P. Blencowe, and K. C. Schwab, Phys. Rev. Lett. 88, 148301 (2002); W. Marshall, C. Simon, R. Penrose, and D. Bouwmeester, ibid. 91, 130401 (2003).

[7] S. Mancini, V. Giovannetti, D. Vitali, and P. Tombesi, Phys. Rev. Lett. 88, 120401 (2002).

[8] E. Buks and M. L. Roukes, J. Microelectromech. Syst. 11, 802 (2002).

[9] J. Eisert and M. B. Plenio, Int. J. Quant. Inf. 1, 479 (2003).

[10] K. Zyczkowski, P. Horodecki, A. Sanpera, and M. Lewenstein, Phys. Rev. A 58, 883 (1998); J. Eisert and M. B. Plenio, J. Mod. Opt. 46, 145 (1999); J. Eisert, Ph.D. thesis, Potsdam, 2001; G. Vidal and R. F. Werner, Phys. Rev. A 65, 032314 (2002); K. Audenaert, M. B. Plenio, and J. Eisert, Phys. Rev. Lett. 90, 027901 (2003).

[11] The $Q$ factor specifies the number of radians of oscillations necessary for the energy to decrease by $1 / e$.

[12] K. Schwab (private communication).

[13] I. Wilson-Rae, P. Zoller, and A. Imamoglu, Phys. Rev. Lett. 92, 075507 (2004).

[14] W. H. Zurek, Rev. Mod. Phys. 75, 715 (2003); D. Giulini et al., Decoherence and the Appearance of a Classical World in Quantum Theory (Springer, New York, 1996).

[15] Therefore, we have not modeled decoherence by merely appending local terms reflecting decoherence in position $\partial_{t} \rho(t)=-i[H, \rho(t)]+\sum_{k=1}^{N} \epsilon_{k}\left[q_{k},\left[q_{k}, \rho(t)\right]\right]$ to the generators of the dynamical map with suitable $\epsilon_{k}>0$ (corresponding to the approximation of the exact generator in Ref. [16] in the limit of very weak damping, very high temperatures, Ohmic spectral density, and factorizing initial conditions), which is not necessarily appropriate, due to the nonfactorizing initial conditions.

[16] B. L. Hu, J. P. Paz, and Y. Zhang, Phys. Rev. D 45, 2843 (1992).

[17] D. A. Harrington, P. Mohanty, and M. L. Roukes, Physica (Amsterdam) 284B, 2145 (2000).

[18] A. N. Cleland and M. L. Roukes, J. Appl. Phys. 92, 2758 (2002).

[19] C. M. Caves et al., Rev. Mod. Phys. 52, 341 (1980).

[20] K. L. Ekinci, Y.T. Yang, X. M. H. Huang, and M. L. Roukes, Appl. Phys. Lett. 81, 3879 (2002).

[21] M. P. Blencowe and M. N. Wybourne, Appl. Phys. Lett. 77, 3845 (2000).

[22] M. D. LaHaye, O. Buu, B. Camarota, and K. C. Schwab, Science 304, 74 (2004).

[23] M. B. Plenio, J. Hartley, and J. Eisert, New J. Phys. 6, 36 (2004). 